\title{
Image Excitons and Plasmon-Exciton Strong Coupling in 2D Perovskite Semiconductors
}

\author{
Wendy Niu, ${ }^{1, *}$ Lindsey A. Ibbotson, ${ }^{1}$ David Leipold,${ }^{2}$ Erich \\ Runge, ${ }^{2}$ G. Vijaya Prakash, ${ }^{3}$ and Jeremy J. Baumberg ${ }^{1, \dagger}$ \\ ${ }^{1}$ NanoPhotonics Centre, Cavendish Laboratory, \\ University of Cambridge, CB3 OHE, UK \\ ${ }^{2}$ Institut für Mikro- und Nanotechnologien, \\ Technische Universität Ilmenau, 98693 Ilmenau, Germany \\ ${ }^{3}$ Nanophotonics lab, Department of Physics, \\ Indian Institute of Technology Delhi, New Delhi, 110016, India
}

(Dated: February 8, 2015)

\begin{abstract}
We present evidence for 'image-biexcitons' within organic-inorganic perovskite-coated silver gratings. These composite quasiparticles are formed by the interaction between an exciton and its image in the metal mirror below, with binding energy $100 \mathrm{meV}$ at room temperature. By changing the polar and azimuthal angles of incident light, we observe strong coupling between excitons and surface plasmon polaritons on the grating, with Rabi splittings of 150 and $125 \mathrm{meV}$ for the exciton and biexciton respectively. Detailed analysis of the field polarisations and dipole orientations shows how these Rabi couplings arise from the strongly compressed field volume.
\end{abstract}

PACS numbers: 42.79.Dj, 71.35.-Gg, 71.36.+c, 73.20.Mf, 78.67.Pt 
Metal halide based organic-inorganic perovskite semiconductors have attracted a great deal of attention for their optical and electrical properties ${ }^{1-13}$. 3D perovskites have recently been used to produce solar cells with efficiencies of up to $15 \%^{1-4}$, while $2 \mathrm{D}$ perovskites are known to form self-assembled multiple quantum well (MQW) systems suited for optoelectronics ${ }^{5-13}$. The structure of a typical 2D lead iodide $(\mathrm{PbI})$ perovskite is shown in Fig. 1(a), consisting of alternating layers of corner-sharing $\mathrm{PbI}_{6}$ octahedra and interdigitating $\mathrm{RNH}_{3}$ molecules (where $\mathrm{R}$ is an organic moeity). Excitons are formed and trapped in the inorganic layers, where the reduction in dimensionality (quantum confinement) and low refractive index organic layers (dielectric confinement) lead to binding energies in excess of $200 \mathrm{meV}^{5}$. Therefore such perovskite semiconductors exhibit strong excitons at room temperature as seen in the sharp strong absorption and photoluminescence peaks [Fig. 1(b)]. These materials are easily processed from solution ${ }^{6}$ and can thus be incorporated into a variety of nanostructures. In addition, due to their high binding energy and oscillator strength, these perovskites are ideal candidates for the production of new mixed light-matter states at room temperature as a result of strong coupling ${ }^{7-12}$.

Recently there has been much interest in the mixed states of excitons and surface plasmon polaritons (SPPs), where Rabi splittings of up to $650 \mathrm{meV}$ have been reported ${ }^{14-21}$. SPPs are collective electron oscillations that travel along a metal-dielectric interface, leading to large local electric field enhancements. Such hybrid exciton-plasmon states can combine the optical nonlinearity of excitons with the field enhancement shown in SPPs. However it is not possible to directly excite SPPs on a metal film due to energy and momentum conservation, so a method such as grating coupling is required ${ }^{22}$. Periodic structures can supply the missing wavevector in units of $G_{m}=2 m \pi / D$ (where $D$ is the periodicity), allowing SPPs to couple to incoming/outgoing photons. To first order, two types of modes can be seen in the spectra of plasmonic gratings: 'photonic' modes caused purely by interference from the periodicity of the structure, and 'plasmonic' modes where SPPs also interact with the diffracted light.

Here we describe the interaction between grating modes and excitons in silver gratings overcoated with the perovskite $\left(\mathrm{C}_{6} \mathrm{H}_{9} \mathrm{C}_{2} \mathrm{H}_{4} \mathrm{NH}_{3}\right)_{2} \mathrm{PbI}_{4}$ (CHPI). We show that selected SPP modes can strongly couple with the exciton for particular angles of incident light, with interaction strengths one order larger than for III-V quantum wells, and at room temperature. We also show that the exciton mode is spectrally split by Coulomb coupling with its im- 
(a)

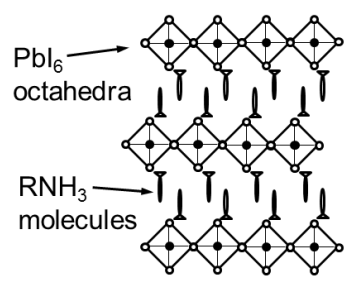

(c)

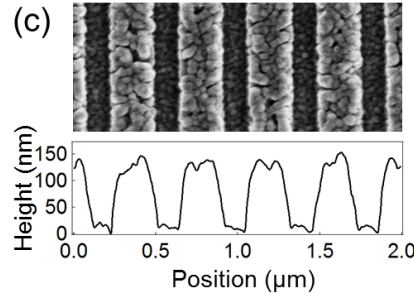

(b)

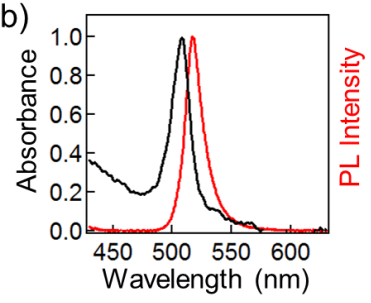

(d)

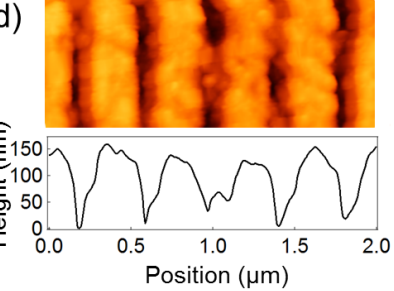

(e)

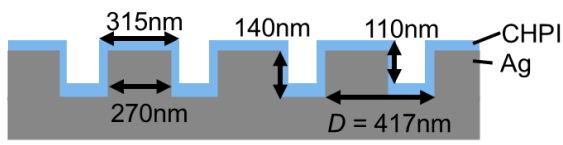

FIG. 1. (a) Schematic of 2D lead iodide perovskite structure. (b) Absorption and photoluminescence spectra of CHPI thin film on glass substrate at room temperature. (c) SEM image (top) and AFM profile (bottom) of Ag grating. (d) AFM image (top) and profile (bottom) of CHPI-coated grating. (e) Schematic cross section of CHPI-coated Ag grating structure.

age in the metallic mirror on which it sits. These results show the promise of such hybrid semiconductors for strong light-matter interactions.

Gratings of periodicity $D=417 \mathrm{~nm}$ are fabricated in ethylene tetrafluoroethylene (ETFE) from nanopatterned silicon stamps using nanoimprinting. An optically opaque Ag layer ( $\sim 120 \mathrm{~nm}$ thick) is deposited onto the polymer to form metal gratings. Chemically synthesised CHPI powder ${ }^{12}$ is dissolved in tetrahydrofuran and spin coated onto the Ag gratings to produce a conformal coating with thickness $\sim 25 \mathrm{~nm}$. Measurements by SEM and AFM of the Ag and CHPI-coated gratings are shown in Figs. 1(c,d) respectively, which allow the dimensions to be extracted as shown in the schematic of the semiconductor-coated structure in Fig. 1(e). Specular reflection measurements are made as a function of the incident polar $(\theta)$ and azimuthal $(\phi)$ angles using a broadband white light source $(215-2500 \mathrm{~nm})$. The sample properties are uniform over $\mathrm{cm}^{2}$ areas, with small variations due to the depth and morphology of the coatings.

TM polarised reflectivity scans of a CHPI-coated Ag grating at $\phi=0^{\circ}$ [Fig. 2(a)] show two dispersionless exciton modes at 480 and $500 \mathrm{~nm}$ (marked by arrows) far off resonance 


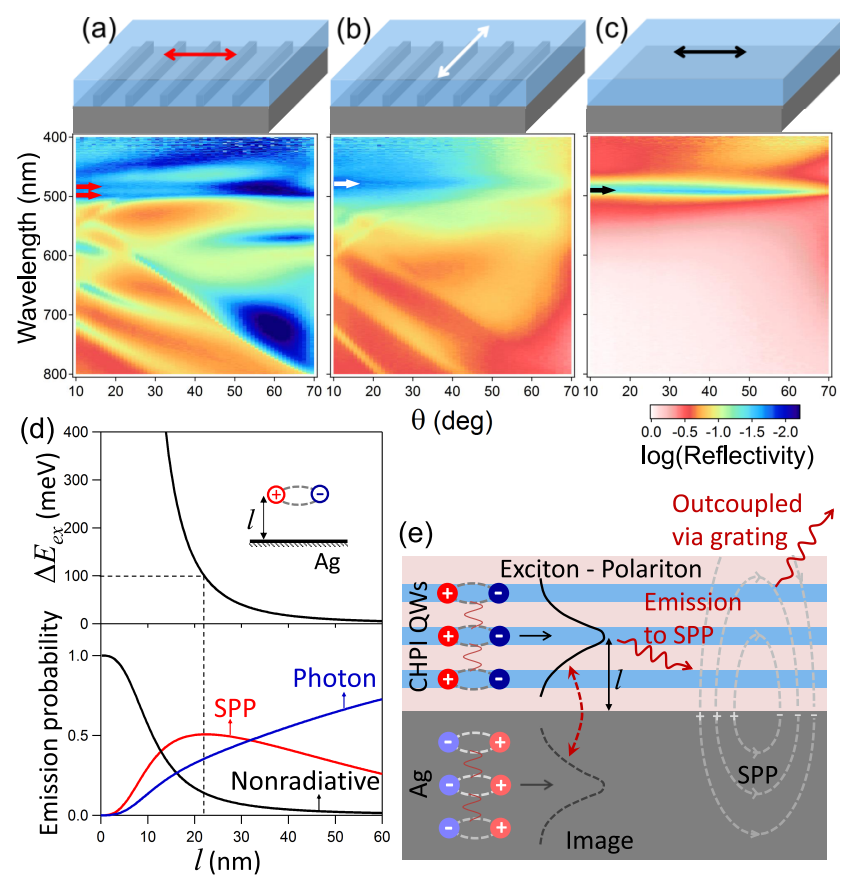

FIG. 2. Specular reflectivity scans at $\phi=0^{\circ}$ : CHPI-coated Ag grating with (a) TM and (b) TE polarised light, and (c) CHPI-coated $120 \mathrm{~nm}$ planar Ag film with TM polarised light. The electric field orientation is shown above each scan, and positions of exciton modes indicated by arrows. (d) Change in emitted energy (top) and relative decay probabilities (bottom) of an exciton with energy $2.6 \mathrm{eV}$ placed distance $l$ from the Ag surface. The dashed line indicates the experimentally measured redshift. (e) Schematic mechanism for SPP-mediated emission of image-biexciton.

with grating modes. The persistent presence of a second exciton is only detected when SPPs can be excited, i. e. in TM polarisation [Fig. 2(a)] but not TE [Fig. 2(b)], nor in CHPI-coated planar Ag films [Fig. 2(c)]. From Fig. 2 we see that SPP excitation leads to the observation of an additional redshifted exciton with a splitting of $100 \mathrm{meV}$. Its appearance only when SPPs are present rules out any influence from modified CHPI assembly in the grooves, which are in any case hundreds of times larger than the PbI layer spacing. In addition the exciton diffusion length in 2D perovskites is of order $10 \mathrm{~nm}^{23}$, therefore we do not expect any limiting effects due to the grating geometry. We note slight changes in the CHPI coverage alter the positions and intensities of dispersive grating modes [ $c f$ Fig. 3(a), with a thinner CHPI coating], however the exciton modes remain essentially unchanged.

It is well known that the emitted energy of a dipole (exciton) is lowered when placed in front of a metallic surface due to interactions between the dipole and the reflected elec- 
tromagnetic field ${ }^{24-29}$. Using the method of images, we can replace the metal and describe instead the coupling between an exciton in the CHPI $\left(\epsilon_{1}\right)$ and its image exciton in the metal $\left(\epsilon_{2}\right)$, modified by their respective dielectric environments. These spin-coated CHPI QWs are universally parallel to the substrate surface as shown by X-ray diffraction ${ }^{6,12}$, and Chance et $a .^{27}$ showed the redshift in the emitted energy of an exciton $\left(\Delta E_{e x}\right)$ oriented parallel to the interface can be approximated by

$$
\Delta E_{e x} \sim\left(\frac{1}{k_{1} l}\right)^{3} \operatorname{Re}\left\{\frac{\epsilon_{2}-\epsilon_{1}}{\epsilon_{2}+\epsilon_{1}}\right\} q \Gamma_{0},
$$

where $l$ is the distance between the exciton and a metal surface, $k_{1}$ is the wavenumber of light in CHPI, $q$ is the quantum yield of CHPI excitons (taken here to be 1 ), and $\Gamma_{0}$ is the inverse exciton radiative lifetime without the metal. Similar to the appearance of excitons in the spectra, we expect to observe such coupled 'image-biexcitons' as minima in the reflectivity, at a wavelength that differs from the uncoupled exciton according to Eq. 1. The strength of coupling between the exciton and reflected electromagnetic field depends on the exciton dipole moment, which is controlled by the term $q \Gamma_{0}$. From this we can see the $l^{-3}$ dependence of the redshift as shown in Fig. 2(d), where the experimentally observed $\Delta E_{\text {ex }} \sim 100 \mathrm{meV}$ corresponds to $l \sim 22 \mathrm{~nm}$, close to the experimentally-determined CHPI thickness. Clearly $\Delta E_{e x}$ is also affected by the dielectric response of CHPI and Ag, and from Eq. 1 we see that $\Delta E_{e x}$ is maximised if $\epsilon_{2}+\epsilon_{1} \rightarrow 0$, i. e. when emission is resonant with an SPP on the metal-dielectric interface. The linewidth of of the exciton is also affected by interactions with image charges in the metal, however in our perovskite system this effect is not dominant due to tight planar confinement of excitons. We expect larger effects in systems that are less perfectly 2D, such as semiconductor heterostructures and J-aggregate systems, where surface charges play a much larger role.

The role of the SPP in this case is to outcouple the signal of the redshifted exciton. There are three main decay channels for dipole emission near a metal surface: direct emission to photons, emission to SPPs, and nonradiative processes such as the excitation of electron-hole pairs and lossy surface waves on the metal. Other nonradiative paths via defects or phonons are independent of $l$ and will be ignored in this analysis. Emission into SPPs provides an extra radiative decay channel as this signal can be extracted to the far field via the periodic nanostructure, and this mechanism has been used to improve the luminescence efficiency of light emitting devices ${ }^{30,31}$. The relative decay probability for each process is calculated as 
a function of $l^{29}$ and shown in Fig. 2(d). Although these calculations are intended for SPPs propagating on planar metal surfaces, we can use them as approximations for our grating system, although we note such estimates are indeed expected to become less accurate with increasing structure depth. Up to a CHPI thickness of $25 \mathrm{~nm}$, SPP mediated emission is the most important radiative decay channel with a maximum emission probability at $22 \mathrm{~nm}$, matching the experimentally observed $\Delta E_{\text {ex }}$. Even for thicker CHPI films we expect the exciton modes to remain at the same positions, because SPP emission becomes weak at large $l$ where $\Delta E_{e x}$ is negligible.

In practice, we observe a range of biexciton energies in our spectra [Figs. 2,3]. There is a clear overall decrease in the reflectivity compared to Ag-only gratings [Supp. Info.] for the wavelength range $490-550 \mathrm{~nm}$, which coincides with the redshifted energies we would expect for the excitons in our $25 \mathrm{~nm}$ film according to Eq. 1. However in all cases we still see the strongest signature from excitons that are $15-25 \mathrm{~nm}$ from the Ag interface, which have the largest SPP emission probability [Fig. 2(d)]. This corresponds to a wavelength of $490-510 \mathrm{~nm}$, where we observe our second dip. Furthermore, the lineshape of the biexciton resonance does not resemble that of a single oscillator, which is due to the superposition of excitons from this range of distances.

In our MQW perovskite system, localised excitons in periodically-spaced nearby QWs are optically coupled together to form many collective exciton-polariton states, each of which has an average distance $l$ from the Ag surface ${ }^{32-35}$. Therefore in CHPI-coated Ag gratings we observe both in-plane exciton-polaritons, and out-of-plane interactions that lead to imagebiexcitons, which are outcoupled via SPP emission with a binding energy of $100 \mathrm{meV}$ at room temperature [Fig. 2(e)]. For our grating system, the exciton and SPP modes become closer in energy with increasing $\phi$ [see below and Fig. 3], and as a result splitting between the exciton modes (indicated by arrows in Fig. 3) increases to around $185 \mathrm{meV}$ at $\phi=90^{\circ}$. The azimuthal dependence of the exciton splitting reflects the tunable modification of the Coulomb interaction in this geometry, but however requires further theoretical development.

Besides the strong excitons, more dispersive grating modes can also be seen in the TM reflectivity scans of CHPI-coated Ag gratings [Fig. 3]. Due to momentum and energy conservation, the dispersion of such grating modes (see Supp. Info.) is given by

$$
k_{m}^{2}=k_{i}^{2} \sin ^{2} \theta+G_{m}^{2} \pm 2 k_{i} G_{m} \sin \theta \cos \phi,
$$




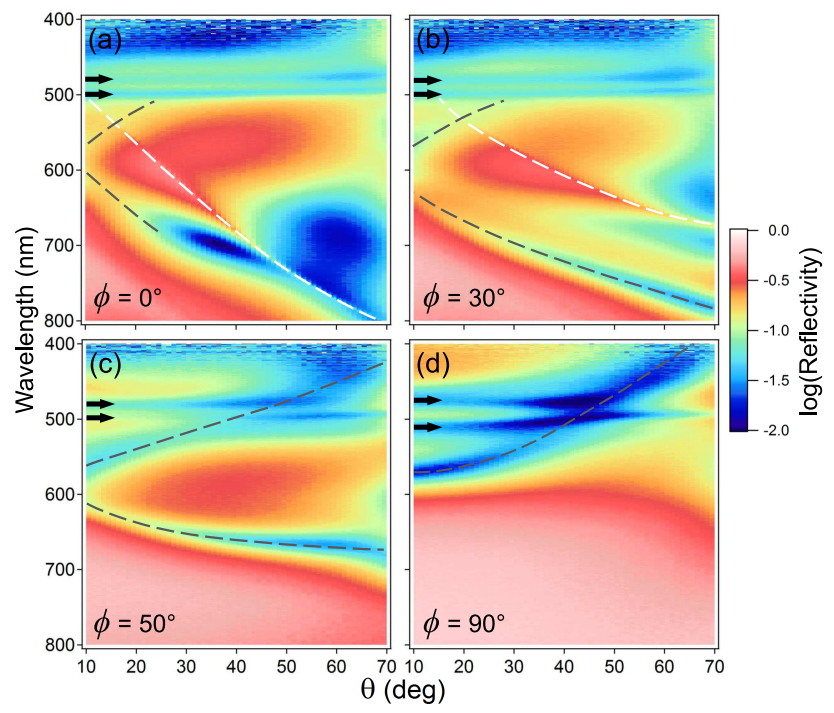

FIG. 3. TM polarised specular reflectivity of CHPI-coated Ag grating, $D=417 \mathrm{~nm}$. White ('photonic' grating modes) and grey dashed lines ('plasmonic' grating modes) are guides, and exciton modes indicated by black arrows. The exciton splitting increases with $\phi$.

where $k_{m}$ is the wavevector of the measured grating mode, and $k_{i}$ is the wavevector of the incident light taking into account the refractive index of $\mathrm{CHPI}^{36}$. In our spectra we observe the $m= \pm 1$ plasmonic modes (grey dashed lines), and as these become resonant with the exciton and image exciton, the light-matter modes strongly couple and produce an anticrossing in the reflectivity of $0.25 \mathrm{eV}$. Extracting the mode positions from the $\phi=90^{\circ}$ scan [Fig. 3(d)] allows them to be fit to a three oscillator model using the Hamiltonian

$$
\hat{H}=\left(\begin{array}{ccc}
E_{e x} & 0 & \Omega_{e x} / 2 \\
0 & E_{b x} & \Omega_{b x} / 2 \\
\Omega_{e x} / 2 & \Omega_{b x} / 2 & E_{p l}
\end{array}\right),
$$

where $E_{e x}, E_{b x}$ and $E_{p l}$ are the energies of the exciton-polariton, image-biexciton and plasmonic grating modes respectively, while $\Omega_{e x}$ and $\Omega_{b x}$ represent the interaction between the SPP and exciton/image-biexciton. From this we find Rabi splittings of $\Omega_{e x}=150 \mathrm{meV}$ and $\Omega_{b x}=125 \mathrm{meV}$. These are greatly enhanced because of the large confinement of the plasmonic optical field in the thin PbI QW layers. The Rabi splitting is given by $\Omega \propto$ $\sqrt{f_{\text {osc }} N_{Q W} / V}$, where the oscillator strength $\left(f_{\text {osc }}\right)$ of the CHPI is assumed to be similar for coupling to photons or plasmons, the number of QWs $\left(N_{Q W}\right)$ is proportional to the CHPI thickness, and the mode volume $(V)$ is here proportional to the optical mode size. Comparing 
to Fabry-Perot planar CHPI microcavities in strong coupling ${ }^{12}$ which have CHPI thickness of $72 \mathrm{~nm}$, cavity length of $407 \mathrm{~nm}$, and a Rabi frequency of $\Omega_{F P}=65 \mathrm{meV}$, the simple scaling above predicts $\Omega_{S P P} \sim \Omega_{F P} \sqrt{(22 / 72) .(407 / 22)}=156 \mathrm{meV}$, in excellent agreement with our measurements. Using SPPs to strongly couple to the excitons thus dramatically reduces the cavity length, thus enhancing the light-matter coupling. We note that in contrast to this scaling between cavity- and plasmonic-enhancements, comparable Rabi splittings are produced by J-aggregate layers on both arrays of Ag holes and inside microcavities, ${ }^{21}$ because field confinements are not similarly concentrated.

We calculate the full eigenstates of the system using finite element method simulations. These confirm the anticrossings observed, and provide the optical field profiles. In the case of strong coupling at $\phi=90^{\circ}$, the time-averaged near-field shows strongest intensity inside the CHPI which coats the bottom surface of the grating, with a rapid evanescent decay away from the interface [Figs. $4(\mathrm{~b}, \mathrm{c})]$. The mode is thus both laterally confined by the grating as well as being trapped inside the surface layers where it couples to the excitons.
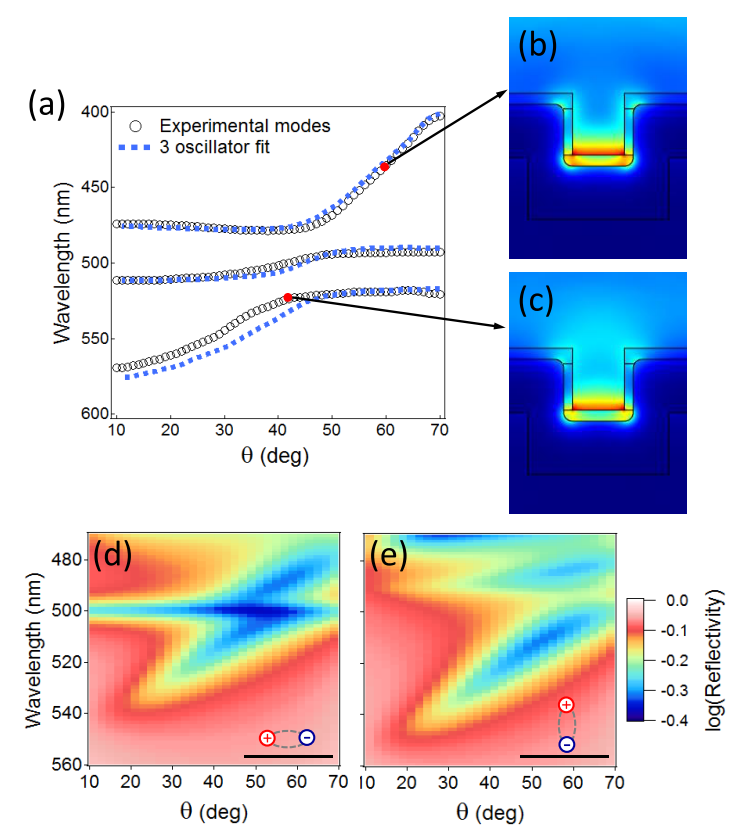

FIG. 4. (a) Extracted spectral mode positions for $\phi=90^{\circ}$ reflection dips (open circles), and fit from three oscillator coupling model (dashed lines). (b,c) Time-averaged $E$-field intensity profiles $(\vec{E} \cdot \vec{E})$ as indicated. (d,e) Simulated reflection spectra for (d) in-plane and (e) out-of-plane exciton dipoles. 
Although CHPI is known to align planar to any local surface, it is possible that coverage may be different on vertical and horizontal surfaces of the grating. However the strongest SPP field intensity is found at the bottom grating surface. Here the SPP E-field direction is primarily perpendicular to the metal-dielectric interface, while excitons in CHPI QWs are polarised parallel to this interface, which is indeed the same as films spin coated onto a flat substrate ${ }^{6,12}$. Simulated $\phi=90^{\circ}$ spectra for in- and out-of-plane exciton dipoles are shown in Figs. 4(d,e) respectively. While strong coupling is seen for both dipole orientations, the bare exciton is only seen for the in-plane dipole. It thus appears that the coupling between the excitons and their images are responsible for mixing the dipole orientations, enabling the strong coupling with the SPP mode. Far-field light is directly coupled into the layered perovskite system, where the excitons mediate SPP interactions. The polariton states mix excitons within the perovskite which are delocalised across many PbI monolayers, with SPPs which are tightly confined to the CHPI layer above the Ag grating and laterally localised in the grating slits by the coupling of standing waves. Such light-matter polaritonic quasiparticles thus combine organic, inorganic and plasmonic components in an unusual fashion.

Strong coupling has previously been observed between inorganic or organic excitons and $\mathrm{Au}$ nanoslit gratings at low temperature. The coupling constants in these systems are much smaller compared to CHPI at room temperature: $55 \mathrm{meV}$ for $50 \mathrm{~nm}$ J-aggregate films at $77 \mathrm{~K}^{16}$, and $8 \mathrm{meV}$ for $10 \mathrm{~nm}$ GaAs QWs at $10 \mathrm{~K}^{15}$. More recently room-temperature strong coupling has been seen for J-aggregate film overcoated Ag hole arrays, with splittings exceeding $600 \mathrm{meV}$ at room temperature due to their large oscillator strengths ${ }^{21}$. One of the key challenges is to produce long-lifetime devices from both CHPI- and J-aggregate nanostructures, which although both stable over many months at room temperature, age over longer times which thus limits current applications. Additionally electrical-pumping which is required in many device applications has now been achieved in perovskite LEDs, which benefit from the layered and well-ordered sheets of CHPI interacting to form welldefined exciton-polaritons. Another key difference with traditional semiconductors is that for III-V semiconductors the QWs have to be spaced at least $20 \mathrm{~nm}$ from the metal surface to maintain their optical quality. In contrast our $25 \mathrm{~nm}$ thick CHPI film is prepared directly on the metal, and still gives strongly radiative exciton modes because the organic sandwich protects the PbI QW layers. Theoretically Fig. 2(d) shows that excitons remain radiative 
via SPP coupling for film thickness above $10 \mathrm{~nm}$. Hence the perovskite system is well suited to manipulate light-matter interactions.

In conclusion, we report evidence of image-biexcitons in perovskite-coated silver gratings with binding energies of $100 \mathrm{meV}$ at room temperature. Such quasiparticles arise from the interaction between excitons and their images in the metal, and are outcoupled from the grating structure via SPP emission. These out-of-plane biexciton states mediate coupling between in-plane QW excitons and out-of-plane SPP grating modes. This enables the observation of strong coupling at room temperature with Rabi splittings of 150 and $125 \mathrm{meV}$ for the exciton and image-biexciton respectively. Such modification of exciton behaviour is of great interest for other layered van der Waals semiconductors such as derivatives of graphene and transition metal dichalcogenides, particularly for future optoelectronic devices that demand large field enhancements by coupling to SPPs.

\section{ACKNOWLEDGMENTS}

This work is part of the UK-India Education Research Initiative (UKIERI) and supported by EPSRC Grants EP/G060649/1, EP/H027130/1, and ERC LINASS 320503.

* wwn20@cam.ac.uk

$\dagger$ jjb12@cam.ac.uk

1 H. S. Kim, C. R. Lee, J. H. Im, K. B. Lee, T. Moehl, A. Marchioro, S. J. Moon, R. HumphryBaker, J. H. Yum, J. E. Moser, M. Grätzel, and N. G. Park, Scientific Reports 2, 591 (2012).

2 M. M. Lee, J. Teuscher, T. Miyasaka, T. N. Murakami, and H. J. Snaith, Science 338, 643 (2012).

3 J. H. Heo, S. H. Im, J. H. Noh, T. N. Mandal, C. S. Lim, J. A. Chang, Y. H. Lee, H. J. Kim, A. Sarkar, M. K. Nazeeruddin, M. Grätzel, and S. I. Seok, Nature Photonics 7, 486 (2013).

4 M. Liu, M. B. Johnston, and H. J. Snaith, Nature 501, 395 (2013).

5 T. Ishihara, Journal of Luminescence 60-61, 269 (1994).

6 D. B. Mitzi, Chemistry of Materials 13, 3283 (2001).

7 T. Fujita, Y. Sato, T. Kuitani, and T. Ishihara, Physical Review B 57, 12428 (1998). 
8 K. Sumioka, H. Nagahama, and T. Tsutsui, Applied Physics Letters 78, 1328 (2001).

9 A. Brehier, R. Parashkov, J. S. Lauret, and E. Deleporte, Applied Physics Letters 89, 171110 (2006).

10 C. Symonds, J. Bellessa, J. C. Plenet, a. Brehier, R. Parashkov, J. S. Lauret, and E. Deleporte, Applied Physics Letters 90, 091107 (2007).

11 G. Lanty, A. Bréhier, R. Parashkov, J. S. Lauret, and E. Deleporte, New Journal of Physics 10, 065007 (2008).

12 K. Pradeesh, J. J. Baumberg, and G. V. Prakash, Optics express 17, 22171 (2009).

13 Z. Cheng and J. Lin, CrystEngComm 12, 2646 (2010).

14 J. Dintinger, S. Klein, F. Bustos, W. Barnes, and T. Ebbesen, Physical Review B 71, 035424 (2005).

15 P. Vasa, R. Pomraenke, S. Schwieger, Y. Y. I. Mazur, V. Kunets, P. Srinivasan, E. Johnson, J. Kihm, D. Kim, E. Runge, G. Salamo, and C. Lienau, Physical Review Letters 101, 116801 (2008).

16 P. Vasa, R. Pomraenke, G. Cirmi, E. De Re, W. Wang, S. Schwieger, D. Leipold, E. Runge, G. Cerullo, and C. Lienau, ACS Nano 4, 7559 (2010).

17 R. DeglInnocenti, S. Zanotto, a. Tredicucci, G. Biasiol, and L. Sorba, Solid State Communications 151, 1725 (2011).

18 S. Balci, C. Kocabas, S. Ates, E. Karademir, O. Salihoglu, and A. Aydinli, Physical Review B 86, 235402 (2012).

19 A. Salomon, R. Gordon, Y. Prior, T. Seideman, and M. Sukharev, Physical Review Letters 109, $073002(2012)$.

20 J. Bellessa, C. Symonds, K. Vynck, a. Lemaitre, a. Brioude, L. Beaur, J. Plenet, P. Viste, D. Felbacq, E. Cambril, and P. Valvin, Physical Review B 80, 033303 (2009).

21 T. Schwartz, J. a. Hutchison, C. Genet, and T. W. Ebbesen, Physical Review Letters 106, 196405 (2011).

22 S. Maier, Plasmonics: Fundamentals and Applications, 2007th ed. (Springer, 2007).

23 S. Ahmad, J. J. Baumberg, and G. Vijaya Prakash, Journal of Applied Physics 114, 233511 (2013).

24 H. Morawitz, Physical Review 187, 1792 (1969).

25 H. Morawitz and M. Philpott, Physical Review B 10, 4863 (1974). 
26 R. R. Chance, A. Prock, and R. Silbey, The Journal of Chemical Physics 60, 2184 (1974).

27 R. R. Chance, A. Prock, and R. Silbey, Physical Review A 12, 1448 (1975).

28 R. R. Chance, A. Prock, and R. Silbey, The Journal of Chemical Physics 62, 2245 (1975).

29 G. Ford and W. Weber, Physics Reports 113, 195 (1984).

30 J. Frischeisen, Q. Niu, A. Abdellah, J. B. Kinzel, R. Gehlhaar, G. Scarpa, C. Adachi, P. Lugli, and W. Brütting, Optics Express S1, A7 (19).

31 A. Kumar, R. Srivastava, P. Tyagi, D. Mehta, and M. Kamalasanan, Organic Electronics 13, $159(2012)$

32 R. Tamaki, Y. Arai, D. Ichikawa, M. Inoue, H. Kunugita, and K. Ema, Journal of Luminescence 128, $842(2008)$

33 J. Baumberg, a. Heberle, a. Kavokin, M. Vladimirova, and K. Köhler, Physical Review Letters 80, 3567 (1998).

34 A. Kavokin and J. Baumberg, Physical Review B 57, 12697 (1998).

35 M. Vladimirova, E. Ivchenko, and A. Kavokin, Semiconductors 32, 90 (1998).

36 See Supplementary Material at for TM polarised specular reflectivity of $D=417 \mathrm{~nm}$ Ag grating. 